\title{
Lupus vulgaris mimicking cutaneous leishmaniasis: A case report
}

\section{Nouf Faihan Bin Rubaian', Haya Fahad Alzamami', Gadah Abdulatif Alhosawi², Leena Abdulrahman Almuhaish ${ }^{3}$}

${ }^{1}$ Dermatology demonstrator at Department of Dermatology, College of Medicine, Imam Abdulrahman bin Faisal University, Dammam, Saudi Arabia, ${ }^{2}$ Dermatology resident at Abqaiq General Hospital, Saudi Arabia, ${ }^{3}$ Intern at College of Medicine, King Fahd Hospital of the University, Khobar, Imam Abdulrahman bin Faisal University, Dammam, Saudi Arabia

Corresponding author: Nouf Faihan Bin Rubaian, MD, E-mail: nbinrubaian@iau.edu.sa

\begin{abstract}
Lupus vulgaris $(\mathrm{LV})$ is a progressive, chronic form of cutaneous tuberculosis (CTB). The head and neck regions are the most commonly affected sites, followed by the arms and legs. Occurring in unusual sites may pose diagnostic difficulties. Herein, we report a case of $L V$ present on the dorsal aspect of the right hand in a twenty-year-old Saudi male. It was misdiagnosed as leishmaniasis as the patient lived in an area in which it was endemic, and was treated accordingly with no benefit. A skin punch biopsy was taken and the diagnosis of LV was confirmed. The lesion responded well to anti-tubercular therapy (ATT), yet healed with atrophic scarring. Although rare, clinicians must be aware of the importance of considering CTB as an important differential, as misdiagnosis or delayed diagnosis of this entity may eventually cause prolonged morbidity.
\end{abstract}

Keywords: Cutaneous tuberculosis; Lupus vulgaris; Cutaneous leishmaniasis; Hands; Al-Ahsa

\section{INTRODUCTION}

Tuberculosis (TB) is defined as an infectious disease caused by the bacterium Mycobacterium tuberculosis. It most commonly affects the lungs, and then is known as pulmonary TB. It may also affect other internal and external organs, and then is known as extrapulmonary TB. This accounts for $14 \%$ of cases of $\mathrm{TB} ; 1-2 \%$ of these are cutaneous TB (CTB) involving the skin [1].

CTB is classified into two major types. The first type is true CTB and is caused by Mycobacterium tuberculosis. It shows a wide variety of clinical manifestations as lesions range from multiple papules seen in primary inoculation tuberculosis and warty-like lesions known as tuberculosis verrucosa cutis to massive ulcers such as Buruli ulcer and plaques found in lupus vulgaris $(\mathrm{LV})$. The second type of CTB is caused by atypical mycobacterium species $[2,3]$.
$\mathrm{LV}$ is one of the clinical manifestations of CTB occurring in individuals sensitized to Mycobacterium tuberculosis [1]. It has been discovered in 1865 by Erasmus Wilson. It is common among children and adolescents, with a prevalence of 41-68\% [3]. LV tends to spread contagiously or via hematogenous or lymphatic spread. It may affect individuals who have received Bacillus Calmette-Guérin (BCG) inoculation or who have a positive delayed-type hypersensitivity reaction to tuberculin due to intact cell-mediated immunity [4]. There are five types of LV: Plaque, hypertrophic or vegetating, tumor-like, papular and nodular, and lastly the ulcerative type, which is known to be the most deformative type of LV [2].

This case report describes a condition of a young male patient complaining of a solitary erythematous hyperkeratotic papule on the dorsal aspect of the right hand, which had been grown slowly and had formed an ulcerative plaque, although this is not a common site of cutaneous TB.

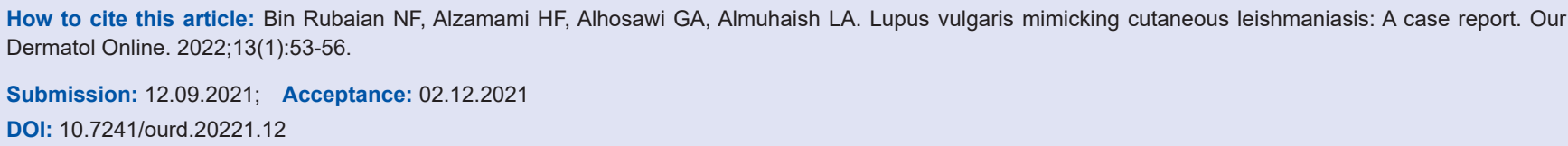




\section{CASE REPORT}

A twenty-year-old Saudi male originally from Al-Ahsa, Eastern Province of Saudi Arabia, not known to have a chronic medical illness, presented to the dermatology clinic at King Fahad Hospital of the University in Khobar with a history of an ulcerated skin lesion on the right hand present for four years. The lesion started as a single asymptomatic papule on the dorsum of the right hand and gradually enlarged and progressed into a plaque with a central ulceration. The patient did not recall a history of insect bites or trauma at the site. He denied a history of fever, night sweats, weight loss, cough, or shortness of breath.

A cutaneous examination revealed a well-defined, verrucous, irregularly bordered, erythematous, scaly plaque, approx. $4 \times 5 \mathrm{~cm}$ in size, with central ulceration and yellowish crustations, involving the dorsum of the right hand (Fig. 1).

The patient intensively sought medical advice in Saudi Arabia and Bahrain. The lesion was treated under the impression of leishmaniasis, given the fact that he was from an area in which the disease was endemic and the lesion was ulcerative. In addition, as the patient was not consistently following a single clinic, but was seen in numerous different clinics, the lesion was treated with various methods, including sodium stibogluconate, topical salicylic acid, and several cryotherapy sessions with no benefit as the diagnosis remained unconfirmed.

Afterward, the patient decided to seek medical advice in India, where investigations were done. Laboratory findings were normal. Chest X-ray was normal. A skin biopsy was taken with hematoxylin and eosin (H\&E) staining. Histopathology revealed tuberculoid granulomatous infiltrate made from lymphocytes, plasma cells, histiocytes, and epithelioid cells with Langhans giant cells, with the overlying epidermis showing a moderate spongiotic psoriasiform change and the underlying dermis showing fibroplasia. Findings were consistent with LV. Images from histopathology could not be taken and only a descriptive report was handed to the patient.

Ultimately, the patient was treated successfully with a four anti-tubercular drug regimen, including isoniazid (INH), rifampicin, ethambutol, and pyrazinamide. The patient received the treatment for nine months yet noted that the lesion healed after only two months of therapy, leaving an atrophic scar at the site of ulceration (Fig. 2).

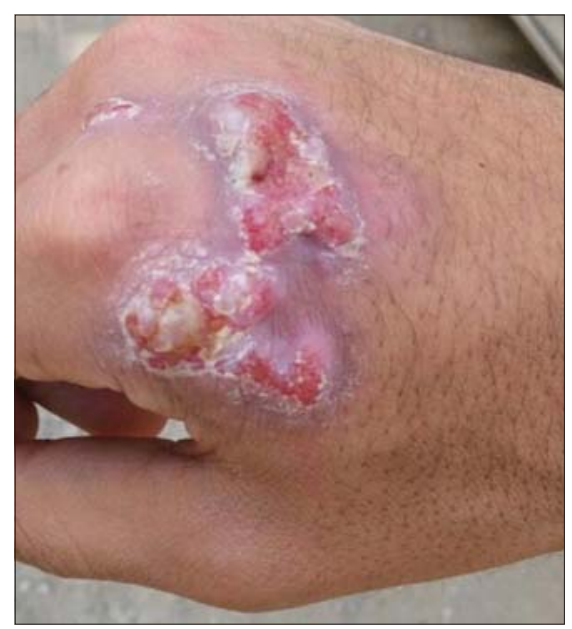

Figure 1: A $4 \times 5 \mathrm{~cm}$, erythematous, scaly plaque with central ulceration on the dorsum of the right hand.

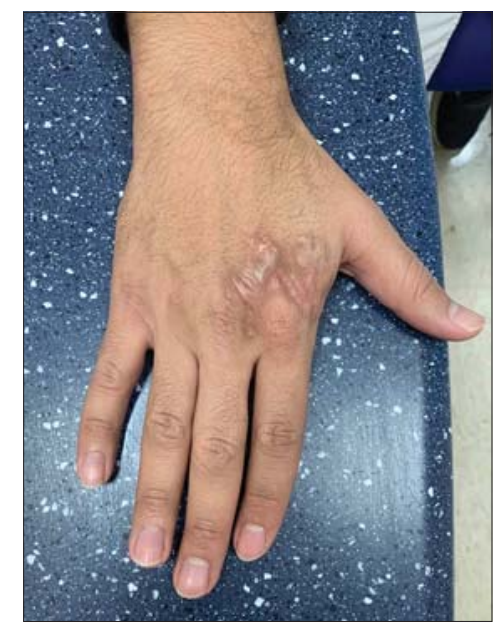

Figure 2: The lesion after it had healed, with atrophic scarring on the site of ulceration.

\section{DISCUSSION}

According to the World Health Organization (WHO), TB is considered the ninth leading cause of death worldwide [2]. Developing countries have the greatest burden of the disease, accounting for more than $90 \%$ of cases [4]. In Saudi Arabia, Riyadh and Dammam have the highest prevalence of Mycobacterium tuberculosis, with $22 \%$ and $21 \%$, respectively, while the prevalence is the lowest in Jazan and Hail, with an incidence of $2 \%$ and $3 \%$, respectively [5].

Tubercular infections vary from pulmonary to extrapulmonary. Only 1.5\% of extrapulmonary cases of $\mathrm{TB}$ show cutaneous involvement [4]. The source of cutaneous a TB infection may be exogenous, endogenous, or following a BCG vaccination [6]. 
LV is a chronic, progressive disease, occurring in patients sensitized to Mycobacterium tuberculosis with moderate to high immunity [6]. The infection occurs mainly through direct extension from the underlying affected tissue or by hematogenous or lymphatics spread. Infection may also occur due to the reactivation of a latent cutaneous focus secondary to previous silent bacteremia in patients in whom the underlying focus is not clear [6]. LV commonly involves the head and neck areas, followed by the arms and legs [7]. In Europe, $80 \%$ of cases involve the head and neck, in particular, the nose and cheeks. On the other hand, in India, the buttocks, thighs, and legs are commonly involved. Usually, A lesion of LV presents itself as a solitary, reddish-brown papule or nodule, soft in consistency, gradually enlarging into a plaque with a tendency to ulcerate. The plaque often shows an apple-jelly color on diascopy [4]. Clinically, LV shows five major clinical patterns depending on the local tissue response to the infection, including plaque, hypertrophic or vegetation, tumor-like, papular or nodular, and the ulcerative type [6]. Plaque-type $L V$ is considered a common form, while based on a study done in India, the ulcerative type was the least common clinical variant, accounting for $14.2 \%$ of cases [8]. Long-standing ulcerative $L V$ may eventually lead to scarring and deformity in addition to the risk of squamous cell carcinoma [9]. Fortunately, our patient had no destruction or deformity in the underlying tissue. However, the lesion healed leaving an atrophic scar.

In our case, the site of involvement considered an exposed area was rarely to be affected. In addition, the lesion greatly mimicked chronic cutaneous leishmaniasis due to the presence of an ulcer, especially since it was endemic in the region from which the patient came. Therefore, it is crucial for clinicians, especially in endemic areas, to keep in mind other differential diagnoses even if the lesions are present in unusual sites. In this case, other than leishmaniasis, CTD, lupus erythematosus, sarcoidosis, and granuloma annulare should definitely be considered [10].

To diagnose $\mathrm{LV}$, there are different modalities that have to be considered. Firstly, a diascopy examination in the clinic will show apple-jelly nodules in the plaques of $\mathrm{LV}$. Secondly, a tuberculin test and interferon-gamma (IFN- $\gamma$ ) release assays (QuantiFERON TB Gold, T-SPOT.TB) may confirm the diagnosis of $\mathrm{LV}$ through the presence of IFN- $\gamma$ from T cells in the patient's blood, mediated by Mycobacterium tuberculosis peptides [11]. Thirdly, a skin biopsy will show the histopathological features of $\mathrm{LV}$, including a thin, atrophied epidermis or acanthosis with hyperkeratosis and pseudoepitheliomatous hyperplasia, as well as scant caseation of tubercles and invisible bacilli [4]. In comparison, the histological features of leishmaniasis are similar to some extent to $\mathrm{LV}$, except for necrosis, atrophy, and hyperplasia of the epidermis. Also, there is a multitude of multinucleated giant cells with slight parasites present in lesions of leishmaniasis. Additionally, amastigote organisms may be visible with H\&E or Giemsa stain. Fourthly, a polymerase chain reaction (PCR) test may also be helpful in diagnosing $\mathrm{LV}$, which will detect positive Mycobacterium tuberculosis organisms $[1,11]$.

Our patient was diagnosed with LV through a skin punch biopsy. The histopathology report revealed patchy, nodular, tuberculoid, granulomatous infiltrate made from lymphocytes, plasma cells, histiocytes, and epithelioid cells with occasional Langhans giant cells. The overlying epidermis showed moderate acanthotic and spongiotic psoriasiform changes. The underlying dermis showed fibroplasia. Chest X-ray, complete blood count, and chemistry were normal.

An appropriate treatment may assist in decreasing the transmission of Mycobacterium tuberculosis and prevent bacterial resistance to antibiotics [3]. Various drugs were highlighted by the WHO: INH, rifampicin, ethambutol, pyrazinamide, and streptomycin [3].

In CTB, the management plan consists of two periods. During the first eight weeks, intense therapy is employed, consisting of INH, rifampicin, ethambutol, and pyrazinamide. Then, therapy is resumed as a maintenance for sixteen weeks with INH and rifampicin. Ethambutol may be used as an alternative to INH in the second phase of the treatment if the patient shows resistance [3].

Our patient was mistreated as a case of leishmaniasis in different dermatology clinics, based on history taking and clinical examination. Sodium stibogluconate injection, topical salicylic acid, and cryotherapy were administered yet the results were unsatisfactory. In the end, the patient underwent anti-tubercular therapy (ATT) and four drugs were prescribed. A remarkable improvement was observed after two months of therapy. The treatment successfully ended after nine months.

\section{CONCLUSION}

An awareness of various clinical manifestations of CTB especially in uncommon sites, as in the 
presented case, is crucial to the early diagnosis and treatment, thus reducing morbidity. In our case, the lesion was misdiagnosed based on clinical findings as leishmaniasis. After a skin biopsy was taken, the diagnosis of $\mathrm{LV}$ was confirmed and ATT was initiated. Afterward, the lesion subsided leaving an atrophic scar in the site of ulceration, which could have been avoided by an early diagnosis.

\section{Consent}

The examination of the patient was conducted according to the principles of the Declaration of Helsinki. The authors certify that they have obtained all appropriate patient consent forms, in which the patients gave their consent for images and other clinical information to be included in the journal. The patients understand that their names and initials will not be published and due effort will be made to conceal their identity, but that anonymity cannot be guaranteed.

\section{REFERENCES}

1. Gunawan H, Achdiat PA, Hindritiani R, Essary ED, Ningtias LD, Siregar EP, et al. Various cutaneous tuberculosis with rare clinical manifestations: A case series. Int J Mycobacteriol. 2018;7:288-91.

2. Chen Q, Chen W, Hao F. Cutaneous tuberculosis: A great imitator. Clin Dermatol. 2019;37:192-9.

3. van Zyl L, du Plessis J, Viljoen J. Cutaneous tuberculosis overview and current treatment regimens. Tuberculosis (Edinb). 2015;95:629-38.

4. Nair PA, Mehta MJ, Patel BB. Ulcerative lupus vulgaris over nose, leading to cosmetic deformity. Indian J Dermatol. 2015;60:104.

5. Al Watban AZ, Al Salamah AA, El Faki MG. Prevalence of suspected tuberculosis in the Kingdom of Saudi Arabia according to conventional and molecular methods. J Family Community Med. 2014;21:182-5

6. Pai VV, Naveen KN, Athanikar SB, Dinesh US, Divyashree A, Gupta G. A clinico-histopathological study of lupus vulgaris: A 3-year experience at a tertiary care centre. Indian Dermatol Online J. 2014;5:461-5.

7. Hassan I, Ahmad M, Masood Q. Lupus vulgaris: An atypical presentation. Indian J Dermatol Venereol Leprol. 2010;76:180-1.

8. Chhangte MZ, Thakur BK, Verma S, Dey B. Ulcerative lupus vulgaris primarily involving the ear lobule. Indian Dermatol Online J. 2019;10:729-31.

9. De D, Khadwal A, Singhi P, Dogra S, Radotra BD, Kanwar AJ. Chronic nonhealing ulcer on the hand. Clin Exp Dermatol. 2009;34:647-8

10. Douba MD, Abbas O, Wali A, Nassany J, Aouf A, Tibbi MS, et al. Chronic cutaneous leishmaniasis, a great mimicker with various clinical presentations: 12 years experience from Aleppo. J Eur Acad Dermatol Venereol. 2012;26:1224-9.

11. Bolognia J, Schaffer J, Cerroni L: Dermatology. $4^{\text {th }}$ ed. Elsevier; 2018.

Copyright by Nouf Faihan Bin Rubaian, et al. This is an open-access article distributed under the terms of the Creative Commons Attribution License, which permits unrestricted use, distribution, and reproduction in any medium, provided the original author and source are credited.

Source of Support: Nil, Conflict of Interest: None declared. 